\title{
Nonlesional skin in atopic dermatitis is seemingly healthy skin - observations using noninvasive methods
}

\author{
Adriana Polańska, Aleksandra Dańczak-Pazdrowska, Wojciech Silny, Dorota Jenerowicz, Karolina Olek-Hrab, \\ Agnieszka Osmola-Mańkowska
}

Department of Dermatology, Poznan University of Medical Sciences, Poland

Videosurgery Miniinv 2013; 8 (3): 192-199 DOI: $10.5114 /$ wiitm.2011.33633

\begin{abstract}
Introduction: Atopic dermatitis (AD) is a chronic and relapsing skin disorder, which is characterized by abnormal skin barrier function within the entire skin surface. Several noninvasive bioengineering methods have been commonly used to quantify disease severity. High-frequency ultrasonography (HF-USG) is an important contribution to this field Aim: To evaluate noninvolved skin during the external treatment in relation to involved regions in patients with $A D$ skin using noninvasive methods.

Material and methods: Transepidermal water loss (TEWL), capacitance and erythema assessment and HF-USG were performed in 55 AD patients within 2 regions (involved and uninvolved skin) before and after therapy. The clinical severity of the disease process was based on the eczema area and severity index (EASI) score. A control group consisting of 15 subjects was also included.

Results: On the basis of 4 bioengineering methods our study revealed that uninvolved skin in $A D$ presents subclinical disturbances and significantly changes during therapy. The HF-USG detects inflammation in the upper dermis in $A D$ patients in the form of a hypoechoic band, which may also be observed to a lesser extent within normal-appearing skin.

Conclusions: Nonlesional skin differs significantly from lesional skin in $A D$ and from skin of healthy subjects. Noninvasive methods are able to measure subclinical skin disturbances within normal-appearing skin, which are not evaluated using standard clinical scores. They are objective and may facilitate communication between different research groups.
\end{abstract}

Key words: atopic dermatitis, seemingly healthy skin, transepidermal water loss, capacitance, high frequency ultrasonography, skin ultrasonography.

\section{Introduction}

Atopic dermatitis (AD) is a multifactorial and complex skin disease affecting an increasing number of patients. Despite the numerous studies conducted on its etiopathogenesis, the exact causative factor is still a matter of debate. Both gene-environment interactions and epidermal barrier abnormalities contribute to the considerable clinical heterogenicity of $A D$, which ranges from dry skin to superinfection and severe erythroderma [1-3]. Conducted studies revealed that clinically normal-appearing skin may be characterized by minimal inflammation with the presence of a sparse perivascular T-cell infiltrate. Also Langerhans cells with surface-bound IgE can be observed to a lesser extent than within affected regions

Dr Adriana Polańska, Department of Dermatology, Poznan University of Medical Sciences, 49 Przybyszewskiego St, 60-355 Poznan, Poland, e-mail: adriana-polanska@wp.pl 
[4-7]. Moreover, the feature of disturbed barrier function with increased water evaporation and reduced water content of stratum corneum (SC) within uninvolved skin regions were detected [8-10]. This may lead to the conclusion that the whole skin, even clinically normal-appearing, in $A D$ needs therapeutic intervention and should be under detailed clinician supervision.

In general routine practice the main attention of the physician is drawn to affected skin areas with marked inflammation and the sites of 'healthy' skin are not evaluated. However, they constitute the minimal version of $A D$ and on their basis widespread inflammation may appear. It would seem that there is a need for complete objective assessment of $A D$, mostly in relation to the clinical trials on newly introduced drugs and better communication between different research groups. In this field several noninvasive, reproducible techniques, which allow for quantification of disease severity, can be proposed. Bioengineering methods, including the measurement of transepidermal water loss (TEWL) and water content of SC (skin capacitance, corneometry), are mostly focused on evaluation of skin barrier function [9-15]. Erythema, which is a sign of exacerbation in $A D$, can be measured by two different principles: reflectance or spectroscopy [16]. Since 1970 high-frequency ultrasonography (HFUSG) has been consistently improved and nowadays has also found an application in evaluation of inflammatory diseases. The thickness of the subepidermal hypoechoic band may reflect an inflammatory process with edema or infiltration in the upper parts of the dermis [17-19].

\section{Aim}

The main purpose of this study was the objective assessment of skin barrier function and inflammation within the skin of AD patients. Noninvasive bioengineering methods such as TEWL measurement, corneometry, erythema measurement and HF-USG were performed within active lesions as well as within surrounding skin areas without any signs of eczema and compared to normal skin of healthy controls. These biophysical assessments were obtained during exacerbation of the disease process and after the treatment, because the authors also aimed to evaluate the usefulness of these methods in assessing the effects of different therapies in AD.

\section{Material and methods Participants}

Fifty-five patients with AD (26 women, 29 men), diagnosed in accordance with the Hanifin and Rajka classification, were enrolled in the study. Their age ranged between 8 and 60 years (mean age: $25.9 \pm 11.8$ years). All patients referred to the Department of Dermatology, Poznan University of Medical Sciences, due to the exacerbation of skin lesions in the period from May 2009 to December 2011. In relation to the patient history and clinical examination the decision on the type of therapy was made by the specialist. Forty-five patients with localized lesions underwent topical therapy with the use of calcineurin inhibitors or glucocorticosteroids during the next 3 weeks. The rest, with more severe lesions, were irradiated with UVA1 phototherapy (340-400 nm, GP-24H, Cosmedico, Medical Systems, Germany). Additional treatments were antihistamines and emollients. The detailed summary of applied therapies in the study group is presented in Table I.

The control group consisted of 15 healthy Caucasian volunteers with no symptoms of $A D$ and no signs of chronic skin diseases influencing skin function. Age range was 18-39 years (mean age: $31 \pm 5.2$ years).

All participants provided written informed consent and the institutional review board approved the study.

\section{Clinical evaluation}

To evaluate clinical severity before and after treatment eczema area and severity index (EASI) was assessed. This score evaluates 4 clinical parameters: erythema, induration/population, excoriation and lichenification on a 0-3 scale within 4 defined body regions (head/neck, upper extremities, trunk, lower extremities) [20].

\section{Test procedure}

All AD patients underwent two assessments with a 3 or 4 week interval depending on the type of therapy (before and after therapy). During each visit clinical evaluation was based on EASI score and 4 different noninvasive measurement were performed. Each measurement was carried out twice in the same location before treatment and after its completion for both lesional and nonlesional skin. As lesional skin 
Table I. Characteristics of the study group in relation to the type of therapy

\begin{tabular}{|c|c|c|c|c|c|c|}
\hline Regimen & $\begin{array}{l}\text { Number } \\
\text { of patients }\end{array}$ & Main objectives & $\begin{array}{l}\text { Duration } \\
\text { [weeks] }\end{array}$ & $\begin{array}{c}\text { Mean age } \\
\text { [years] }\end{array}$ & $\begin{array}{c}\text { Women } \\
{[\%]}\end{array}$ & $\begin{array}{c}\text { Men } \\
{[\%]}\end{array}$ \\
\hline tGS & 20 & $\begin{array}{l}\text { Group 5-7 of tGS (twice/day on affected regions), } \\
\text { emollients, second generation antihistamines } \\
\text { (cetirizine } 20 \mathrm{mg} \text { ) }\end{array}$ & 3 & 22.5 & 30 & 70 \\
\hline $\mathrm{tCl}$ & 25 & $\begin{array}{l}\text { Pimecrolimus (twice/day on affected regions), } \\
\text { emollients, second generation antihistamines } \\
\text { (cetirizine } 20 \mathrm{mg} \text { ) }\end{array}$ & 3 & 25.0 & 60 & 40 \\
\hline UVA 1 & 10 & $\begin{array}{l}\text { Cycle of } 20 \text { irradiations with weekend break - } \\
\text { dose range } 30-120 \mathrm{~J} / \mathrm{cm}^{2} \text {, emollients, second } \\
\text { generation antihistamines (cetirizine } 20 \mathrm{mg} \text { ) }\end{array}$ & 4 & 33.0 & 50 & 50 \\
\hline Total & 55 & - & - & 25.9 & 47 & 53 \\
\hline
\end{tabular}

tGS - topical steroids, tCI - topical calcineurin inhibitors, UVA1 - narrow-band UVA phototherapy

the antecubital fossa, which is the predilection site for AD, was selected. Evaluation of nonlesional skin was performed approximately $10 \mathrm{~cm}$ from the active eczema area within the flexural site of the upper extremity. The biophysical measurements were always conducted in the same order: TEWL, corneometry, erythema, HF-USG.

In the control group bioengineering readings were taken only once and were carried out within the flexural site of the upper extremity in an established order.

All measurements were performed in the same room conditions (temperature $20-22^{\circ} \mathrm{C}$, humidity 20 $40 \%$ ) after $15-30$ min acclimatization by the same trained physician.

\section{Evaluation with biophysical methods}

Passive diffusion of water through the SC (TEWL) was measured with Tewameter TM 300 (CourageKhazaka, Köln, Germany) according to the guidelines of the standardization group of the European Society of Contact Dermatitis [10, 11]. At least 20 measurements given as a mean value and expressed in $\mathrm{SI}$ units $\left(\mathrm{g} / \mathrm{m}^{2} / \mathrm{h}\right)$ were carried out. The TEWL values within the normal range were established as $0-25 \mathrm{~g} / \mathrm{m}^{2} / \mathrm{h}$.

Corneometr CM 825 (Courage-Khazaka, Köln, Germany) determined the electrical capacitance moisture. This instrument is designed to assess hydration of SC (corneometry) and its principle is based on the fact that the dielectric constant of water is 81 and that of dry skin is below this. A normal value of SC hydration was accepted as higher than $40 \mathrm{u}$. Five measurements given as a mean value in arbitrary units (range: 0-130) were obtained in accordance with guidelines [10, 12].

Erythema measurement was assessed with Color Meter II (Cortex Technology, Hadsund, Denmark). The device is equipped with different color systems. For this study the erythema index was determined by skin reflectance spectroscopy, were the redness is calculated by subtracting the absorbance due to melanin from the absorbance of the green filter [10, 16]. Three independent measurements were performed at an interval of $30 \mathrm{~s}$, on the basis of which the average value was determined.

The width of the subepidermal hypoechoic band and mean skin echogenicity were measured with a high frequency ultrasound scanner (Dermascan C ver. 3, Cortex Technology, Hadsund, Denmark). This instrument operates at a frequency of $20 \mathrm{MHz}$, with a resolution of $60 \mu \mathrm{m} \times 200 \mu \mathrm{m}$ (axial $\times$ lateral) and approximately $15-\mathrm{mm}$ penetration. The ultrasonic wave is partially reflected at the boundary between adjacent structures and produces echoes of different amplitudes. The intensity of the reflection echoes is evaluated by the microprocessor and is visualized as a color-coded two-dimensional Bmode image (standardized code of 255 levels). The average amplitude of the echoes in a defined area of the image is known as echogenicity, which may be objectively measured with computer-assisted image analysis. Hypoechogenic is defined as an intensity < 30 pixels. In A-mode interfaces are shown as well-defined peaks. A typical gain curve 
was 25-70 dB. The velocity of ultrasound in the skin was set at $1.580 \mathrm{~m} / \mathrm{s}[19,21]$.

Using the program built into the USG system 2 parameters were assessed: skin echogenicity (\%) and the thickness of the hypoechoic band. The mean hypoechoic band width was evaluated in millimeters by measuring the vertical distance between the lower edge of the entry echo and the posterior margin of the hypoechoic zone (A-mode scans). Scans were performed by the same trained observer.

\section{Statistical analysis}

For parameters such as echogenicity, hypoechoic band thickness, TEWL, corneometry, and erythema index appropriate descriptive statistics were determined. The compatibility of these parameters with normal distribution was checked and Bartlett's test of homogeneity of variance was performed. For characteristics consistent with a normal distribution for comparison between groups analysis of variance was used. When compatibility with normal distribution was not confirmed, nonparametric tests, Mann-Whitney or Wilcoxon tests, were used. Calculations were performed with the statistical package Statistica v 10.0.

\section{Results}

\section{Eczema area and severity index}

In all $A D$ patients we observed improvement of clinical status expressed as a reduction of EASI score. The median EASI index value before treatment was 26.6 points (min.: 0.0, max.: 69.5) and at the end of therapy was 13.3 points (min.: 0.0 , max.: 54 ). This difference was statistically significant $(p<0.05)$.

\section{Transepidermal water loss}

The mean TEWL of AD patients was higher before the introduction of therapy than after its completion within lesional $(p<0.05)$ as well as nonlesional skin $(p<0.05)$. Also the mean TEWL of AD patients measured within lesional skin was higher before and after therapy than that of normal controls $(p<0.05)$. However, mean TEWL of AD patients detected within nonlesional skin differed statistically significantly from normal controls only in relation to the measure before treatment $(p<0.05)$. The mean TEWL of AD patients after therapy measured within nonlesional skin was not statistically significantly different from that of normal control. Also differences in mean TEWL of AD patients between lesional and nonlesional skin before treatment and after treatment were analyzed. Statistically significant differences in mean TEWL were obtained $(p<0.05)$. Mean TEWL values are summarized in Table II.

\section{Corneometry}

The mean water hydration of $A D$ patients was statistically significantly higher after treatment within lesional skin $(p<0.05)$, while the statistical analysis did not reveal a significant difference within nonlesional skin of $A D$ patients in this parameter before and after treatment. The mean water hydration of the control group was higher than in AD patients in relation to the measure before and after therapy of lesional skin as well as nonlesional skin. Also differences in mean hydration of $A D$ patients between lesional and nonlesional skin before treatment and after treatment were analyzed. Statistically signifi-

Table II. The results of four selected parameters of skin function (TEWL, capacitance, erythema, skin echogenicity, SLEB) measured in AD patients and in the control group

\begin{tabular}{|lccccc|}
\hline \multirow{2}{*}{ Skin parameter } & \multicolumn{2}{c}{ Atopic dermatitis before therapy } & \multicolumn{2}{c}{ Atopic dermatitis after therapy } & \multirow{2}{*}{ Control group } \\
\cline { 2 - 5 } & Involved skin & Uninvolved skin & Involved skin & Uninvolved skin & \\
\cline { 1 - 4 } TEWL $\left[\mathrm{g} / \mathrm{m}^{2} / \mathrm{h}\right]$ & $33.5 \pm 12.4$ & $17.8 \pm 9.3$ & $21.3 \pm 11.8$ & $13.3 \pm 5.3$ & $13.3 \pm 4.7$ \\
\hline Capacitance $(\mathrm{AU})$ & $19.9 \pm 7.9$ & $28.5 \pm 12.5$ & $26.7 \pm 13.5$ & $29.6 \pm 11.3$ & $44.6 \pm 11.9$ \\
\hline Erythema & $16.7 \pm 3.4$ & $11.3 \pm 2.7$ & $13.8 \pm 3.4$ & $11.6 \pm 2.6$ & $11.0 \pm 1.9$ \\
\hline Skin echogenicity [\%] & $7.8 \pm 0.4$ & $8.3 \pm 0.5$ & $8.1 \pm 0.4$ & $8.4 \pm 0.4$ & $8.8 \pm 0.1$ \\
\hline SLEB $[\mathrm{mm}]$ & $0.23 \pm 0.1$ & $0.14 \pm 0.1$ & $0.16 \pm 0.1$ & 0.0 & 0.0 \\
\hline
\end{tabular}

TEWL - transepidermal water loss, AU - arbitrary units, SLEB - subepidermal low-echogenic band 
cant differences in mean skin hydration were obtained $(p<0.05)$. The results are listed in Table II.

\section{Erythema}

The mean erythema index of $A D$ patients was statistically significantly lower after treatment within lesional skin $(p<0.05)$, while within nonlesional skin it did not differ significantly before and after therapy. The mean erythema index of the control group was statistically lower than in $A D$ patients in relation to the measure before and after therapy of lesional skin $(p<0.05)$. Regarding nonlesional skin of AD patients and control group results, a statistically significant difference was not observed. Also differences in mean erythema index of AD patients between lesional and nonlesional skin before treatment and after treatment were analyzed. Statistically significant differences in mean erythema index were revealed $(p<0.05)$. Results are presented in Table II.

\section{Ultrasonography}

The mean skin echogenicity of $A D$ patients was statistically significantly lower before treatment within lesional skin $(p<0.05)$, while within nonlesional skin it did not differ significantly before and after therapy. The mean skin echogenicity of the control group was significantly higher than in AD patients in relation to the measure before and after therapy of lesional skin as well as in relation to the nonlesional skin $(p<0.05)$. Also differences in mean skin echogenicity of $A D$ patients between lesional and nonlesional skin before treatment and after treatment were analyzed and revealed statistically significant differences $(p<0.05)$. The mean hypoechoic band thickness of AD patients was statistically significantly wider before treatment within lesional skin $(p<0.05)$, while within nonlesional skin this hypoechoic band was detected in 7 patients before treatment and disappeared totally after therapy. In the control group a hypoechoic band was not detected. The mean values of these parameters are listed in Table II.

\section{Discussion}

The introduction of so-called proactive therapy in $A D$ drew the attention of researchers to clinically uninvolved skin $[3,6,7]$. It seems that reproducible and representative methods, which allow one to eliminate the influence of subjective evaluation of disease severity, are in this aspect especially needed.
In our study we used 4 noninvasive methods in the assessment of different external therapies in $A D$, mostly in the context of normal- appearing skin assessment.

Measurement of TEWL is a well-recognized parameter that allows objective assessment of the functional status and homeostasis of the epidermal barrier $[9,10]$. According to available literature data, the evaluation of TEWL is characterized by extraordinary sensitivity in detecting even subclinical disturbances. Proksch et al. reported a fourfold increase in TEWL value measured within lesional skin and its twofold elevation within intact skin of AD patients compared to healthy skin [8]. Another parameter useful to determine skin barrier function is skin capacitance, which reflects water content of SC. Insufficient hydration causes impairment of SC and increases the likelihood of skin irritation [22].

Our observations on the elevated TEWL in AD are consistent with previous reports of other authors $[4,10,23-26]$. The average value of TEWL initially was $33.5 \pm 12.4 \mathrm{~g} / \mathrm{m}^{2} / \mathrm{h}$ and after therapy decreased significantly to $21.3 \pm 11.8 \mathrm{~g} / \mathrm{m}^{2} / \mathrm{h}$. We also considered TEWL of the clinically uninvolved skin and its average value before treatment differed significantly compared to the measurement performed after $3 / 4$ weeks of therapy (from $17.8 \pm 9.3 \mathrm{~g} / \mathrm{m}^{2} / \mathrm{h}$ to $13.3 \pm 5.3 \mathrm{~g} / \mathrm{m}^{2} / \mathrm{h}$ ). What is more, in the control group the average TEWL was significantly lower compared to measurements of $A D$ skin. Skin barrier function of AD patients reached the same status as healthy control skin only within uninvolved regions after therapy.

In regards of skin hydration, we obtained statistically significantly lower mean values of skin capacitance within skin lesions before treatment, compared to the state after its completion $(19.9 \pm 7.9$ and 26.7 \pm 13.5 , respectively). Analyzing the same parameters within normal-appearing skin, we did not observe a statistically significant difference. However, comparing the state of hydration of $A D$ patients with healthy subjects we found statistically lower corneometric values in the study group. Average skin hydration in healthy subjects was $44.6 \pm 11.9$ and was higher compared to every measurement of $A D$ skin. These results are in contradiction to some previous studies. For example, Werner did not find a difference in the level of skin hydration of intact skin of $A D$ patients and healthy subjects [26]. On the other hand, Holm et al. presented results similar to our study [27]. 
Another skin parameter which can be easily measured by noninvasive methods is erythema. Red color of the skin is a sign of inflammation and is the most prominent in the acute stage of the disease. In our study we detected a decrease of skin inflammation after 3/4 weeks of therapy expressed as a reduced average value of erythema. Moreover, we noted that uninvolved skin of $A D$ patients did not show a change in skin color and presented the same value of erythema during the course of therapy. This may indicate that erythema measurement is not as sensitive as the measurement of TEWL and cannot be used to detect subclinical lesions. Beside this, the average value of erythema of uninvolved $A D$ skin was similar to the average value determined in the healthy control group. This observation confirms an earlier statement that erythema is particularly associated with acute inflammation, and its measurement remains consistent with clinical assessment of non-involved skin.

One of the newly introduced methods in the assessment of AD is HF-USG, which is easily reproducible and allows clinicians to visualize pathologic changes of the skin in vivo $[19,28,29]$. Different disorders, depending on their etiology, may significantly influence the ultrasound image by changing its echogenicity [30]. Atopic dermatitis is one of the inflammatory diseases and typical sonographic features of this dermatosis is the presence of a hypoechoic band and a decrease in the average skin echogenicity. According to the literature, a hypoechoic band is also known as a subepidermal low echogenic band (SLEB) and expresses infiltration of inflammatory cells and papillary edema $[19,28,29$, 30]. The SLEB is not characteristic only for $A D$ and was first diagnosed in photodamaged skin, but also may be identified in malignant T-cell lymphomas of the skin [31-35].

We found SLEB within involved AD skin before the therapy in all analyzed patients. Average thickness was then $0.229 \pm 0.12 \mathrm{~mm}$. After $3 / 4$ weeks of therapy we observed its decrease to the mean value of $0.160 \pm 0.08 \mathrm{~mm}$. The reduction of this band may serve as a marker of the efficacy of received therapy, but on the other hand the lack of its complete decrease may indicate the need for further continuation of treatment. Analyzing the ultrasound of uninvolved AD skin we noted the presence of SLEB in 7 patients (12.7\%). Its average thickness was 0.140 $\pm 0.05 \mathrm{~mm}$ and was lower compared to the average thickness of SLEB observed in skin lesions. Earlier studies from our department also revealed SLEB within intact skin in $40 \%$ of respondents [19]. This is a relatively novel observation. Currently there are no literature data to explain this phenomenon. We think that this band may suggest the existence of subclinical (and therefore imperceptible to the naked eye) inflammation, which may lead in future to the development of fully symptomatic skin lesions. From the therapeutic point of view, in such cases the use of proactive therapy seem to be effective.

In the group of $A D$ patients we observed an increase in mean skin echogenicity during the treatment, in both lesional and clinically intact skin. Average echogenicity of the affected skin before treatment was $7.8 \pm 0.4 \%$ and increased significantly to 8.1 $\pm 0.4 \%$. Thus, due to the fact that clinical improvement was observed, increased echogenicity, like decreased SLEB, may indicate a regression of inflammation and reduction of edema/infiltration in the upper layers of the dermis. What is more, the measurement of the non-involved skin echogenicity was higher both before and after 3/4-week therapy than the mean skin echogenicity of affected regions.

In the present study, in sonograms of the control group we observed homogeneous echogenicity of the entire skin, and the lack of SLEB. Average skin echogenicity was $8.8 \%$ and was significantly higher not only compared to the affected skin lesions in $A D$ patients, but also compared to their nonlesional skin.

The application of ultrasound in AD has not been widely discussed in previous studies [28, 36]. Danish researchers reported the presence of a wide SLEB in eczematous skin compared with uninvolved skin and, what is more, about the SLEB in the skin of healthy controls [28]. In our opinion healthy skin cannot be characterized by a SLEB, which reflects, as mentioned above, edema, infiltration with inflammatory or malignant cells, or degeneration of elastin.

In this study we present the complete evaluation of disease severity on the basis of a widely accepted clinical score (subjective assessment) as well as noninvasive methods (objective methods). Our study is the first to attempt to demonstrate the usefulness of four methods in overall evaluation of AD patients during the treatment in relation to clinically uninvolved skin. We have shown how properly performed treatment may change the parameters of skin function, even if it seems that the skin appears healthy. For the first time skin sonography was based on 
measurement of SLEB and skin echogenicity within clinically lesional and intact skin.

The general observations revealed that nonlesional skin of $A D$ patients differs from that of healthy controls in a few aspects and should be referred to as seemingly healthy skin. It is characterized by impaired barrier function, as has been underlined in many studies. We have confirmed the previous reports that TEWL and corneometry may be useful to detect subclinical skin disturbances. The application of HF-USG in this study sheds new light in this aspect. Sonography additionally can reveal the level of edema/inflammatory infiltration in those regions and indicates the patients who need special doctor supervision (e.g. patient-oriented treatment). An undeniable advantage of this method is the possibility to examine the skin at any stage of treatment without any special preparations. This makes USG particularly useful in assessing the effects of therapy in children.

\section{References}

1. Rosińska-Więckowicz A, Czarnecka-Operacz M. Disease extent and severity in patients with atopic dermatitis and food allergy. Postep Derm Alergol 2011; 28: 382-8.

2. Leung DY, Boguniewicz M, Howell MD, et al. New insights into atopic dermatitis. J Clin Invest 2004; 5: 651-7.

3. Bieber T. Atopic dermatitis. Ann Dermatol 2010; 22: 125-37.

4. Finlay AY, Nicholls S, King CS, Marks R. The 'dry' non-eczematous skin associated with atopic eczema. Br J Dermatol 1980; 103: 249-56.

5. Uehara M. Clinical and histological features of dry skin in atopic dermatitis. Acta Derm Venereol 1985; 114: 82-6.

6. Wollenberg A, Bieber T. Proactive therapy of atopic dermatitis an emerging concept. Allergy 2009; 64: 276-8.

7. Silny W, Czarnecka-Operacz M, Gliński W. Atopic dermatitis contemporary view on pathomechanism and management. Position statement of the Polish Dermatological Society specialists. Postep Derm Alergol 2010; 27: 365-83.

8. Proksch E, Fölster-Holst R, Bräutigam M, et al. Role of the epidermal barrier in atopic dermatitis. J Dtsch Dermatol Ges 2009; 10: 899-910.

9. Cork MJ, Danby SG, Vasilopoulos Y, et al. Epidermal barrier dysfunction in atopic dermatitis. J Invest Dermatol 2009; 129: 1892-908.

10. Polańska A, Dańczak-Pazdrowska A, Silny W, et al. Evaluation of selected skin barrier functions in atopic dermatitis in relation to the disease severity and pruritus. Postep Derm Alergol 2012; 29: 373-7.

11. Pinnagoda J, Tupker RA, Agner T, et al. Quidelines for transepidermal water loss measurement: a report from the standardization group of the European Society of Contact Dermatitis. Contact Dermatitis 1990; 22: 164-78.
12. Berardesca E. EEMCO guidelines for the assessment of stratum corneum hydratation; electrical methods. Skin Res Technol 1997; 3: $126-32$.

13. Misiak P, Jabłoński S, Rzepkowska-Misiak B. Evaluation of the effectiveness of thoracic sympathectomy in the treatment of primary hyperhidrosis of hands and armpits using the measurement of skin resistance. Videosurgery Miniinv 2012; 7: 147-55.

14. Wójcik A, Budzisz E, Rotsztejn H. Skin surface lipids and their measurements. Postep Derm Alergol 2011; 28: 498-505.

15. Kepska A, Haftek M, Nosbaum A, et al. Psychological stress and transepidermal water loss in atopic dermatitis: preliminary results. Postep Derm Alergol 2012; 29: 263-6.

16. Fullerton A, Fischer T, Lahti A, et al. Guidelines for measurement of skin colour and erythema. A report from the Standardization Group of the European Society of Contact Dermatitis. Contact Dermatitis 1996; 35: 1-10.

17. Serup J. Characterization of contact dermatitis and atopy using bioengineering techniques. A survey. Acta Derm Venereol Suppl (Stockh) 1992; 177: 14-25.

18. Serup J, Jemec GB. Handbook of noninvasive methods and the skin. CRC Press, Boca Raton 1995.

19. Dańczak-Pazdrowska A, Polańska A, Silny W, et al. Seemingly healthy skin in atopic dermatitis: observations with the use of high-frequency ultrasonography, preliminary study. Skin Res Technol 2012; 18: 162-7.

20. Hanifin JM, Thurston M, Omoto M, et al. The eczema area and severity index (EASI): assessment of reliability in atopic dermatitis. Exp Dermatol 2001; 10: 11-8.

21. Jemec GB, Gniadecka M, Ulrich J. Ultrasound in dermatology. Part I. High frequency ultrasound. Eur J Dermatol 2000; 10: 492-7.

22. Darlenski R, Sassning S, Tsankov N, et al. Non-invasive in vivo methods for investigation of the skin barrier physical properties. Eur J Pharm Biopharm 2009; 72: 295-303.

23. Rajka G. Transepidermal water loss on the hands in atopic dermatitis. Arch Dermatol Forsch 1974; 251: 111-5.

24. Proksch E, Fölster-Holst R, Jensen JM. Skin barrier function, epidermal proliferation and differentiation in eczema. J Dermatol Sci 2006; 43: 159-69.

25. Werner Y, Lindberg M. Transepidermal water loss in dry and clinically normal skin in patients with atopic dermatitis. Acta Derm Venereol 1985; 65: 102-5.

26. Werner Y. The water content of the stratum corneum in patients with atopic dermatitis. Measurement with the Corneometer CM 420. Acta Derm Venereol 1986; 66: 281-4.

27. Holm EA, Wulf HC, Thomassen L, et al. Instrumental assessment of atopic eczema: validation of transepidermal water loss, stratum corneum hydration, erythema, scaling, and edema. J Am Acad Dermatol 2006; 55: 772-80.

28. Jasaitiene D, Valiukeviciene S, Linkeviciute G, et al. Principles of high-frequency ultrasonography for investigation of skin pathology. J Eur Acad Dermatol Venereol 2011; 25: 375-82.

29. Polańska A, Dańczak-Pazdrowska A, Silny W, et al. High-frequency ultrasonography in monitoring the effects of treatment of selected dermatoses. Postep Derm Alergol 2011; 28: 255-60. 
30. Jemec GB, Gniadecka M, Ulrich J. Ultrasound in dermatology. Part I. High frequency ultrasound. Eur J Dermatol 2000; 10: 492-7.

31. Wilczak M, Kampioni M, Szmeja J. Castleman's disease - a case report. Videosurgery Miniinv 2011; 6: 27-32.

32. Olek-Hrab K, Osmola-Mańkowska A, Silny W, et al. Use of UVA1 in the treatment of mycosis fungoides - case report. Postep Derm Alergol 2011; 28: 158-64.

33. Otto M, Wieprzowski Ł, Dzwonkowski J, et al. Castleman's disease - an unusual indication for laparoscopic adrenalectomy. Videosurgery Miniinv 2012; 7: 50-4.

34. Fornage BD. High-frequency sonography of the skin. Eur J Ultrasound 1995; 2: 173-82.

35. Rallan D, Harland CC. Ultrasound in dermatology - basic principles and applications. Clin Exp Dermatol 2003; 28: 632-8.

36. Hoffmann K, Dirschka T, Schwarze H. Non-invasive evaluation of inflammation in atopic dermatitis. J Eur Acad Dermatol Venereol 1994; 3: 347-53.

Received: 22.01.2013, accepted: 2.02.2013. 